\title{
FIVE LAYERED FCC FERROMAGNETIC FILMS AS DESCRIBED BY MODIFIED SECOND ORDER PERTURBED HEISENBERG HAMILTONIAN
}

\author{
P. SAMARASEKARA* AND B. I. WARNAKULASOORIYA
}

Department of Physics, University of Peradeniya, Peradeniya, Sri Lanka

\begin{abstract}
For the first time, the $2^{\text {nd }}$ order perturbed Heisenberg Hamiltonian with all the seven magnetic energy parameters was solved for fcc structured ferromagnetic films with five layers. Previously, the $2^{\text {nd }}$ order perturbed Heisenberg Hamiltonian was solved for four layered ferromagnetic films under some special assumptions by us. However, the exact solution of $2^{\text {nd }}$ order perturbed Heisenberg Hamiltonian without any special assumption is formulated in this manuscript. The magnetic easy and hard directions were determined using the angles corresponding to the energy minimum and maximum in the 3-D and 2-D plots. The shape of the 3-D plots doesn't change with the number of layers except the position of spikes. However, the magnetic easy direction varies with the number of layers. Most of the theoretical data obtained by our model agree with the experimental data of ferromagnetic thin films.
\end{abstract}

Keywords: Heisenberg Hamiltonian, ferromagnetic, thin films, spin

\section{INTRODUCTION}

Ferromagnetic films are prime candidates of magnetic memory and microwave devices. The orientation of easy and hard directions is important in all these applications. The Heisenberg Hamiltonian with few magnetic energy parameters has been employed to explain the ultra thin ferromagnetic films (Hucht \& Usadel 1997, Hucht \& Usadel 1999, Usadel \&

* Corresponding author Email: pubudus@pdn.ac.lk 
Hucht 2002). Theoretical study of domain structure and Magnetization reversal in thin magnetic films has been carried out (Nowak 1995). Dipole coupling anisotropy of a square ferromagnetic Heisenberg monolayer has been theoretically described using Heisenberg Hamiltonian (Dantziger et al. 2002). Magnetic ordering in ferro-antiferromagntic bilayers with interfacial coupling has been explained using the Heisenberg Hamiltonian (Tsai et al. 2003). Surface elastic stress of EuTe films has been theoretically explained using Heisenberg Hamiltonian (Radomska \& Balcerzak 2003). Ni films prepared on Cu have been theoretically described using the Korringa-Kohn-Rostoker Green's function method (Ernst et al. 2000). Electric and magnetic properties of multiferroic thin films have been theoretically investigated by modified Heisenberg model using Green's function technique (Kovachev \& Wesselinowa 2009). The quasistatic magnetic hysteresis of ferromagnetic thin films grown on a vicinal substrate has been theoretically investigated by Monte Carlo simulations within a 2D model (Zhao et al. 2002).

According to our previous experimental studies, the stress induced anisotropy plays a vital role in magnetic thin films (Samarasekara \& Cadieu 2001, Samarasekara 2002, Samarasekara 2003). Ferromagnetic and ferrite thin and thick films have been theoretically explained using unperturbed, $2^{\text {nd }}$ order perturbed and $3^{\text {rd }}$ order perturbed Heisenberg Hamiltonian by us previously (Samarasekara 2006, Samarasekara 2007, Samarasekara \& Silva 2007, Samarasekara 2010, Samarasekara 2011). In our previous research work, the pseudo inverse of the matrix was found manually under the assumption of $\mathrm{C}^{+} \mathrm{C}^{+}=1$ to avoid tedious derivations for ferromagnetic ultra thin films (Samarasekara 2006). This same assumption was applied to determine the energy of thick ferromagnetic (Samarasekara \& Silva 2007, Samarasekara 2010) and ferrite (Samarasekara 2011, Samarasekara \& Mendoza 2011) films by us previously. However, the exact pseudo inverse found using the relationship of $C . C^{+}=1-\frac{E}{N}$ is presented in this report. MATHEMATICA computer software was employed to find the exact solution of $C . C^{+}=1-\frac{E}{N}$ for ferromagnetic films with four and five layers. 


\section{MODEL}

The Heisenberg Hamiltonian of ferromagnetic films can be formulated as following.

$$
\begin{aligned}
\mathrm{H}=- & \frac{J}{2} \sum_{m, n} \vec{S}_{m} \cdot \vec{S}_{n}+\frac{\omega}{2} \sum_{m \neq n}\left(\frac{\vec{S}_{m} \cdot \vec{S}_{n}}{r_{m n}{ }^{3}}-\frac{3\left(\vec{S}_{m} \cdot \vec{r}_{m n}\right)\left(\vec{r}_{m n} \cdot \vec{S}_{n}\right)}{r_{m n}{ }^{2}}\right)-\sum_{m} D_{\lambda_{m}}{ }^{(2)}\left(S_{m}{ }^{z}\right)^{2}-\sum_{m} D_{\lambda_{m}}{ }^{(4)}\left(S_{m}{ }^{z}\right)^{4} \\
& -\sum_{m, n}\left[\vec{H}-\left(N_{d} \vec{S}_{n} / \mu_{0}\right)\right] \vec{S}_{m}-\sum_{m} K_{s} \operatorname{Sin} 2 \theta_{m}
\end{aligned}
$$

Here $\vec{S}_{m}$ and $\vec{S}_{n}$ are two spins. Above equation can be deduced to following form.

$$
\begin{aligned}
\mathrm{E}(\theta)=- & \frac{1}{2} \sum_{m, n=1}^{N}\left[\left(J Z_{|m-n|}-\frac{\omega}{4} \Phi_{|m-n|}\right) \cos \left(\theta_{m}-\theta_{n}\right)-\frac{3 \omega}{4} \Phi_{|m-n|} \cos \left(\theta_{m}+\theta_{n}\right)\right] \\
& -\sum_{m=1}^{N}\left(D_{m}{ }^{(2)} \cos ^{2} \theta_{m}+D_{m}{ }^{(4)} \cos ^{4} \theta_{m}+H_{\text {in }} \sin \theta_{m}+H_{\text {out }} \cos \theta_{m}\right) \\
& +\sum_{m, n=1}^{N} \frac{N_{d}}{\mu_{0}} \cos \left(\theta_{m}-\theta_{n}\right)-K_{s} \sum_{m=1}^{N} \sin 2 \theta_{m}
\end{aligned}
$$

Here $J, Z_{|m-n|}, \omega, \Phi_{|m-n|}, \theta, D_{m}{ }^{(2)}, D_{m}{ }^{(4)}, H_{\text {in }}, H_{\text {out }}, N_{d}, K_{s}, m, n$ and $N$ are spin exchange interaction, number of nearest spin neighbors, strength of long range dipole interaction, constants for partial summation of dipole interaction, azimuthal angle of spin, second and fourth order anisotropy constants, in plane and out of plane applied magnetic fields, demagnetization factor, stress induced anisotropy constant, spin plane indices and total number of layers in film, respectively.

The azimuthal angles of spins can be given as $\theta_{m}=\theta+\varepsilon_{m}$ and $\theta_{n}=\theta+\varepsilon_{n}$. After substituting these new angles in above equation number 1, the cosine and sine terms can be expanded up to the second order of $\varepsilon_{m}$ and $\varepsilon_{n}$ as following.

$E(\theta)=E_{0}+E(\varepsilon)+E\left(\varepsilon^{2}\right)$

Here $E_{0}=-\frac{1}{2} \sum_{m, n=1}^{N}\left(J Z_{|m-n|}-\frac{\omega}{4} \Phi_{|m-n|}\right)+\frac{3 \omega}{8} \cos 2 \theta \sum_{m, n=1}^{N} \Phi_{|m-n|}$

$$
\begin{array}{r}
-\cos ^{2} \theta \sum_{m=1}^{N} D_{m}{ }^{(2)}-\cos ^{4} \theta \sum_{m=1}^{N} D_{m}{ }^{(4)}-N\left(H_{\text {in }} \sin \theta+H_{\text {out }} \cos \theta-\frac{N_{d}}{\mu_{0}}+K_{s} \sin 2 \theta\right) \\
E(\varepsilon)=-\frac{3 \omega}{8} \sin 2 \theta \sum_{m, n=1}^{N} \Phi_{|m-n|}\left(\varepsilon_{m}+\varepsilon_{n}\right)+\sin 2 \theta \sum_{m=1}^{N} D_{m}{ }^{(2)} \varepsilon_{m}+2 \cos ^{2} \theta \sin 2 \theta \sum_{m=1}^{N} D_{m}{ }^{(4)} \varepsilon_{m}
\end{array}
$$




$$
\begin{aligned}
& -H_{\text {in }} \cos \theta \sum_{m=1}^{N} \varepsilon_{m}+H_{\text {out }} \sin \theta \sum_{m=1}^{N} \varepsilon_{m}-2 K_{s} \cos 2 \theta \sum_{m=1}^{N} \varepsilon_{m} \\
E\left(\varepsilon^{2}\right)= & \frac{1}{4} \sum_{m, n=1}^{N}\left(J Z_{|m-n|}-\frac{\omega}{4} \Phi_{|m-n|}\right)\left(\varepsilon_{m}-\varepsilon_{n}\right)^{2}-\frac{3 \omega}{16} \cos 2 \theta \sum_{m, n=1}^{N} \Phi_{|m-n|}\left(\varepsilon_{m}+\varepsilon_{n}\right)^{2} \\
& -\left(\sin ^{2} \theta-\cos ^{2} \theta\right) \sum_{m=1}^{N} D_{m}{ }^{(2)} \varepsilon_{m}{ }^{2}+2 \cos ^{2} \theta\left(\cos ^{2} \theta-3 \sin ^{2} \theta\right) \sum_{m=1}^{N} D_{m}{ }^{(4)} \varepsilon_{m}{ }^{2} \\
& +\frac{H_{\text {in }}}{2} \sin \theta \sum_{m=1}^{N} \varepsilon_{m}{ }^{2}+\frac{H_{\text {out }}}{2} \cos \theta \sum_{m=1}^{N} \varepsilon_{m}{ }^{2}-\frac{N_{d}}{2 \mu_{0}} \sum_{m, n=1}^{N}\left(\varepsilon_{m}-\varepsilon_{n}\right)^{2} \\
& +2 K_{s} \sin 2 \theta \sum_{m=1}^{N} \varepsilon_{m}{ }^{2}
\end{aligned}
$$

After using the constraint $\sum_{m=1}^{N} \varepsilon_{m}=0, E(\varepsilon)=\vec{\alpha} \cdot \vec{\varepsilon}$

Here $\vec{\alpha}(\varepsilon)=\vec{B}(\theta) \sin 2 \theta$ are the terms of matrices with

$$
B_{\lambda}(\theta)=-\frac{3 \omega}{4} \sum_{m=1}^{N} \Phi_{|\lambda-m|}+D_{\lambda}{ }^{(2)}+2 D_{\lambda}{ }^{(4)} \cos ^{2} \theta
$$

Also $E\left(\varepsilon^{2}\right)=\frac{1}{2} \vec{\varepsilon} \cdot C \cdot \vec{\varepsilon}$

Here the elements of matrix are given by,

$$
\begin{aligned}
C_{m n} & =-\left(J Z_{|m-n|}-\frac{\omega}{4} \Phi_{|m-n|}\right)-\frac{3 \omega}{4} \cos 2 \theta \Phi_{|m-n|}+\frac{2 N_{d}}{\mu_{0}} \\
& +\delta_{m n}\left\{\sum_{\lambda=1}^{N}\left[J Z_{|m-\lambda|}-\Phi_{|m-\lambda|}\left(\frac{\omega}{4}+\frac{3 \omega}{4} \cos 2 \theta\right)\right]-2\left(\sin ^{2} \theta-\cos ^{2} \theta\right) D_{m}{ }^{(2)}\right. \\
& \left.+4 \cos ^{2} \theta\left(\cos ^{2} \theta-3 \sin ^{2} \theta\right) D_{m}{ }^{(4)}+H_{\text {in }} \sin \theta+H_{o u t} \cos \theta-\frac{4 N_{d}}{\mu_{0}}+4 K_{s} \sin 2 \theta\right\}
\end{aligned}
$$

Therefore, the total magnetic energy given in equation 2 can be deduced to

$\mathrm{E}(\theta)=\mathrm{E}_{0}+\vec{\alpha} \cdot \vec{\varepsilon}+\frac{1}{2} \vec{\varepsilon} \cdot C \cdot \vec{\varepsilon}=\mathrm{E}_{0}-\frac{1}{2} \vec{\alpha} \cdot C^{+} \cdot \vec{\alpha}$

Here $C^{+}$is the pseudo-inverse given by

$$
\text { C. } C^{+}=1-\frac{E}{N} \text {. }
$$

Here $E$ is the matrix with all elements $E_{m n}=1$. 


\section{RESULTS AND DISCUSSION}

The elements of 4 by 4 matrix $C$ were found first. Then the exact pseudo inverse was found using equation 7 for the Heisenberg Hamiltonian with all seven magnetic energy parameters. Finally total energy was found using equation 6. 3-D plot of total magnetic energy versus stress induced anisotropy and azimuthal angle of spin $(\theta)$ for fcc structured ferromagnetic film with four layers is shown in figure 1. Here the other magnetic energy parameters were kept as following.

$$
\omega=1, D_{m}{ }^{(2)}=30, D_{m}{ }^{(4)}=20, J=10, H_{\text {in }}=H_{\text {out }}=\frac{N_{d}}{\mu_{0}}=10
$$

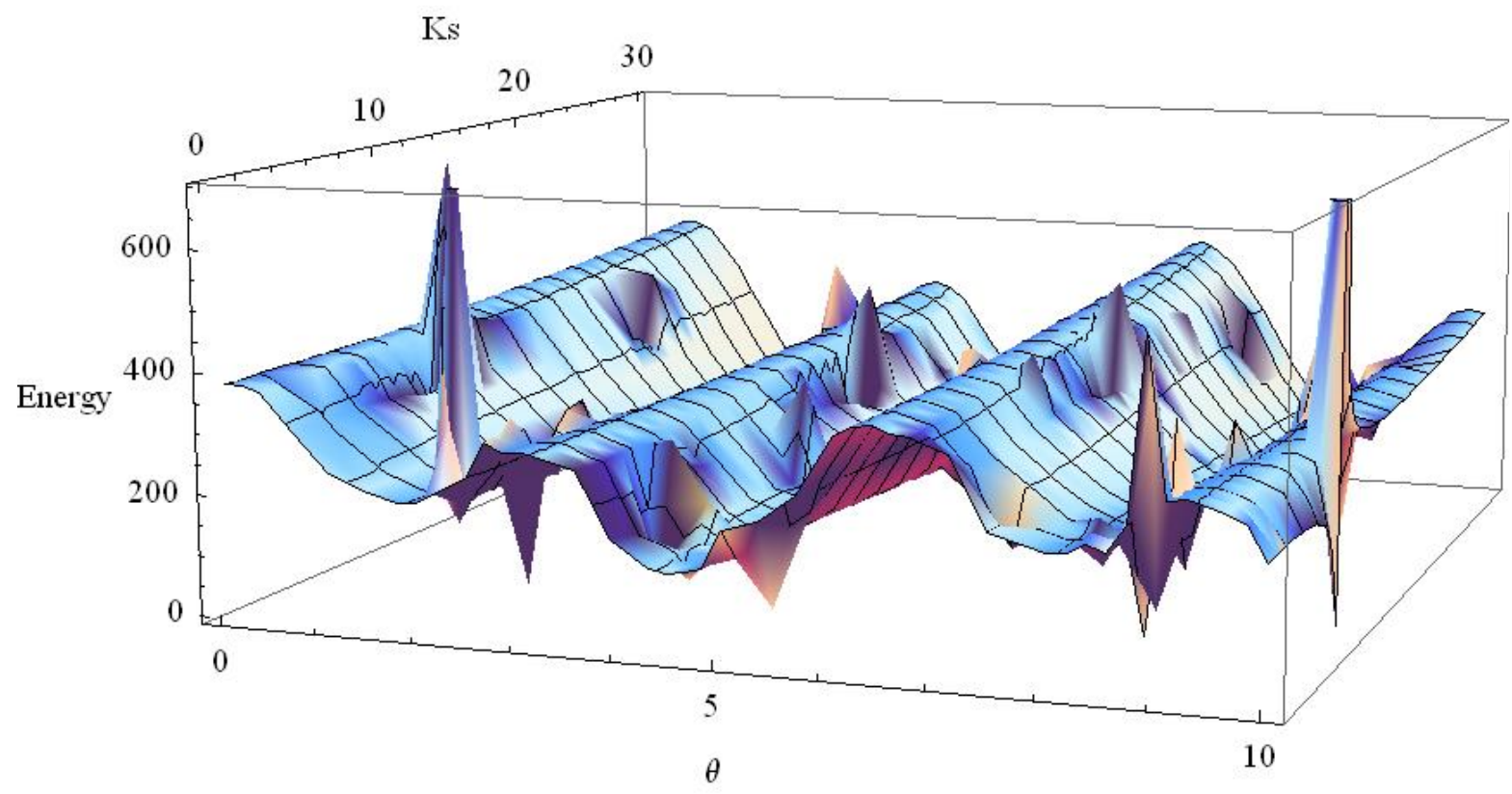

Figure 1: 3-D plot of energy versus $K_{s}$ and $\theta$ for film with four layers

Among several maximums and minimums, one maximum and one minimum of this 3D plot can be observed at $K_{s}=10$ and 14.2, respectively. Figure 2 shows the graph of total magnetic energy versus azimuthal angle for $K_{s}=10$. Easy and hard directions of magnetizations correspond to the energy minimums and maximums of the plot. According to this graph, the hard direction of magnetization can be observed at $0.35,3.30$, ---- radians. Similarly the magnetic easy directions were found to be $2.1,5.0$,---- etc. Spikes appear in the 
graph due to the singularities in the equation of the total magnetic energy. When the pseudo inverse $\left(C^{+}\right)$was found, some terms appeared in the denominator of the equations of $C^{+}$. This was found to be the reason for the singularities in the equation of the total magnetic energy. Previously the Heisenberg Hamiltonian was solved for sc ferromagnetic film with three layers by assuming that the $C^{+}$is the normal inverse of the matrix $C$ (Samarasekara 2006). The 3-D plot of energy versus $K_{s}$ and angle in the previous manuscript is different from the 3-D plot given in Figure 1 in this manuscript. Compared to the plot given in this manuscript, several less spaced peaks could be observed in the same plot presented in the previous manuscript. Furthermore, maximum magnetic energy in that case was higher than that in this manuscript.

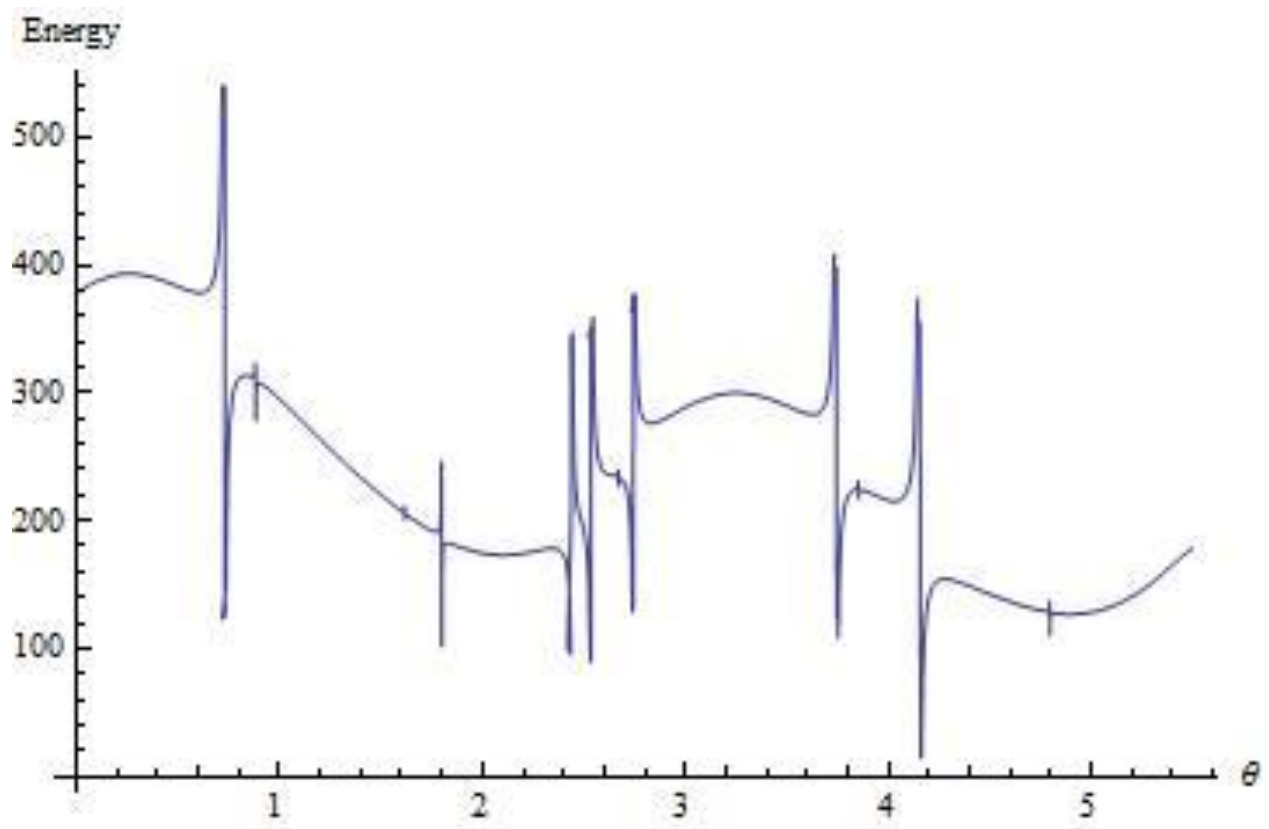

Figure 2: Graph of energy versus angle for $K_{s}=10$

Figure 3 indicates the 3-D plot of the total magnetic energy versus azimuthal angle and stress induced anisotropy for fcc ferromagnetic film with five layers. This graph is similar to the 3-D plot of film with four layers given in figure 1 except the position of spikes. Among several maximums and minimums, one energy minimum and maximum of this graph can be observed at $K_{s}=6.1$ and 23 , respectively. The magnetocystalline anisotropy of a material depends on the spin structure of the material, and it is a unique property of the material. However, the stress induced anisotropy of a thin film sample depends on the deposition condition such as temperature, annealing condition and the physical properties of the substrate 
such as thermal expansion coefficient. When the magnetocrystalline anisotropy of a material is really small, the stress induced anisotropy is comparable to the magnetocrystalline anisotropy. As a matter fact, the stress induces anisotropy governs the magnetic properties (Samarasekara 2001, 2002, 2003). Here the other magnetic energy parameters were kept at $\omega=1, D_{m}{ }^{(2)}=30, D_{m}{ }^{(4)}=20, J=10, H_{\text {in }}=H_{\text {out }}=\frac{N_{d}}{\mu_{0}}=10$

Figure 4 shows the graph of energy versus angle for fcc ferromagnetic film with five layers and $K_{s}=23$. Minimums and maximums of these graphs corresponding to magnetic easy and hard directions are observed at $\theta=2.2,5.2,------$ and $\theta=0.5,3.5,------$, respectively. The angle between the nearest minimum and maximum is approximately 1.7 radians according to figures 2 and 4. This implies that the angle between nearest magnetic easy and hard directions is about 97 degrees. Experimentally the angle between easy and hard direction was found to be 90 degrees for magnetic thin films (Samarasekara 2002). Our theoretical value is really close to the experimental value.

As the number of layers is changed, the easy and hard directions slightly vary according to figures 2 and 4. In addition, the easy axis of magnetization rotates away from the normal drawn to the films plane as the number of layer is increased by indicating a preferred in plane orientation of magnetic easy axis at higher thicknesses. Experimentally, the magnetic easy axis of sputter synthesized fcc structured ferromagnetic Ni films with lattice parameter $0.352 \mathrm{~nm}$ indicates a preferred in plane orientation at higher thicknesses (Parlak 2015). According to this experimental study, the spin reorientation transition from in plane to out plane occurs when the film thickness is in between 14 and $24{ }^{0} \mathrm{~A}$. In addition, the magnetic easy axis of ferromagnetic Fe thin films synthesized by electron beam evaporation rotates from out of plane to in plane direction at the film thickness of 2 monolayers, as the film thickness is increased (Araya-Pochet 1988). For a detailed quantitative theoretical study, the magnetic easy axis orientation has to be theoretically investigated for a wide range of thicknesses from 1 spin layer to many hundred spin layers instead of four to five layers. 


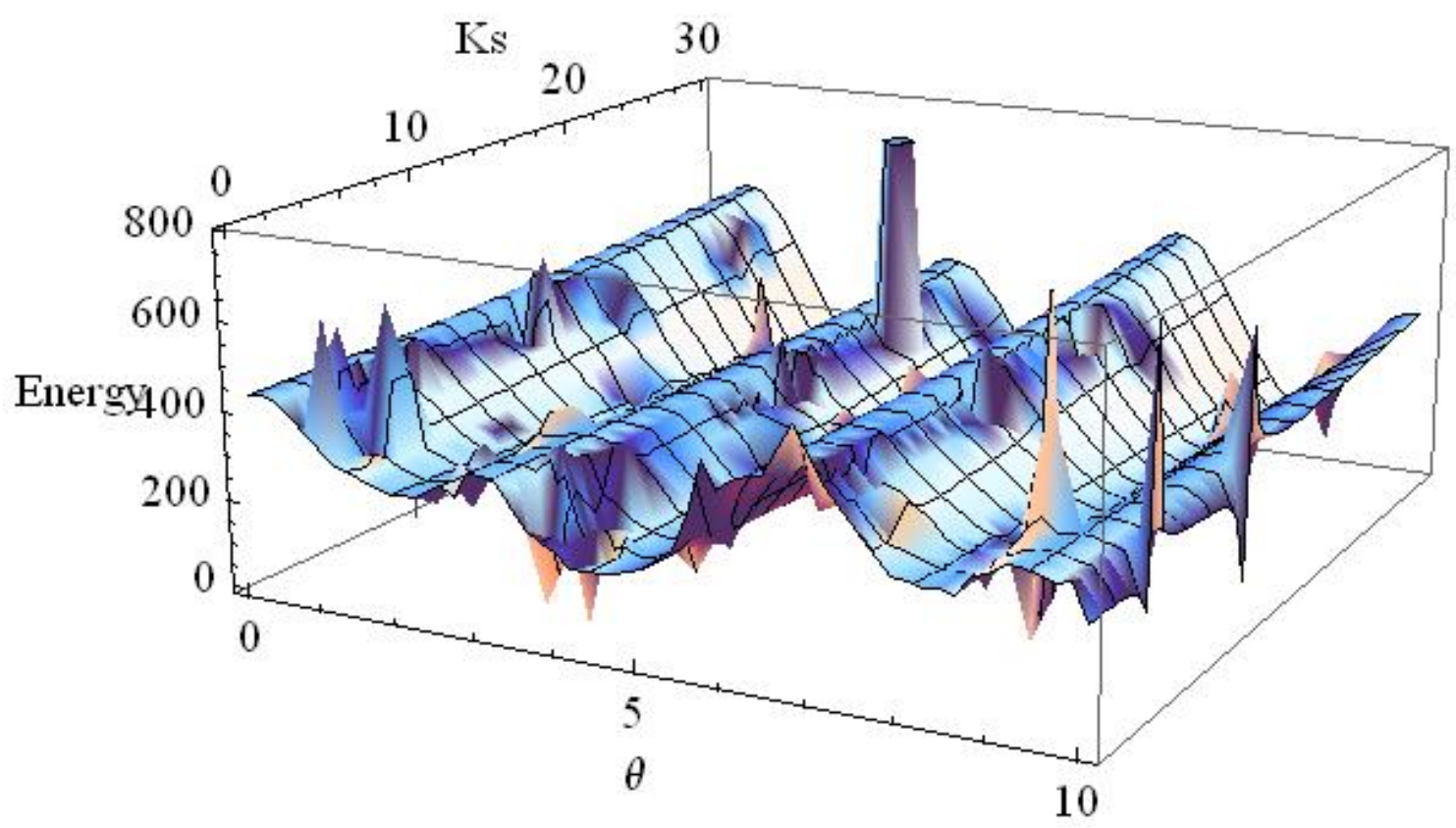

Figure 3: 3-D plot of energy versus angle and $K_{s}$ for a film with five layers

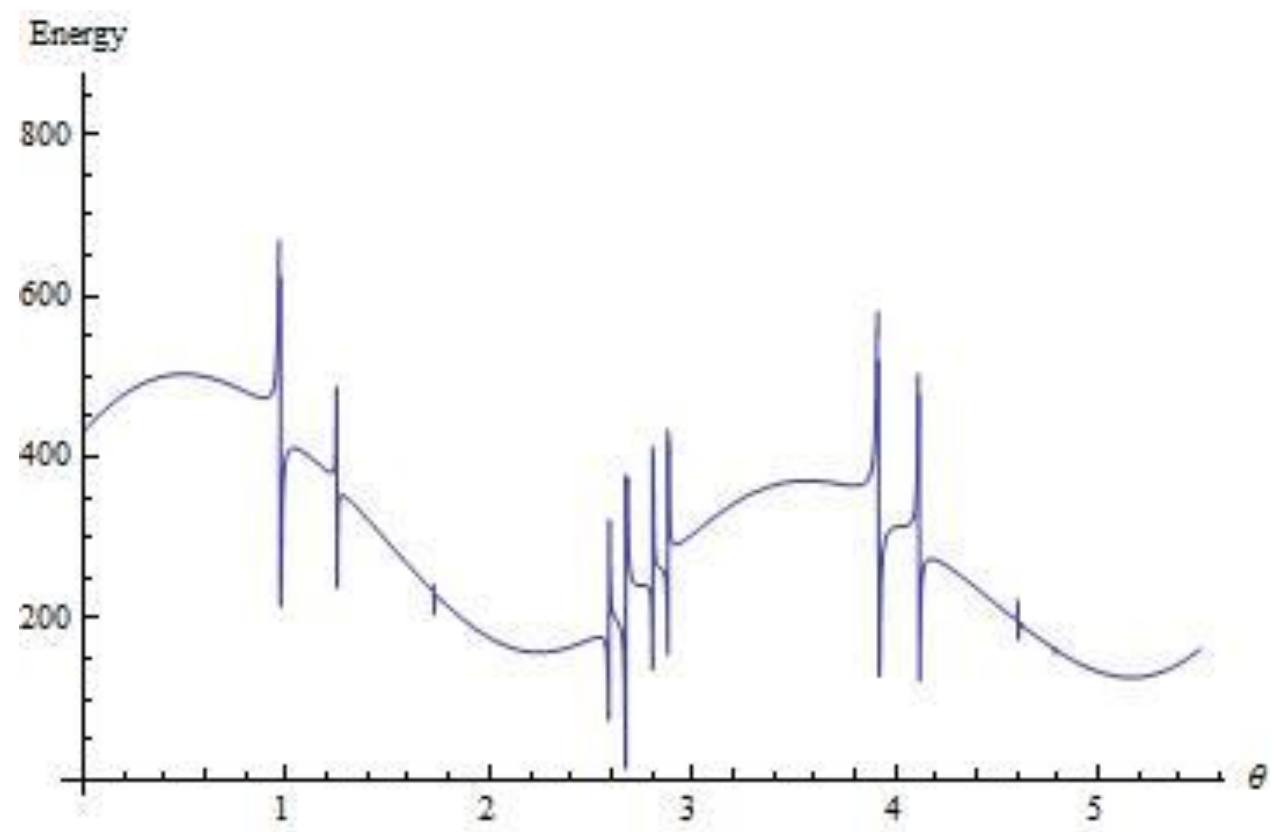

Figure 4: Energy versus angle for $K_{s}=23$ and film with five layers

Figure 5 shows the graph of energy versus azimuthal angle and in plane magnetic field for fcc ferromagnetic film with five layers. One energy minimum and maximum of this graph can be observed at $H_{i n}=5.9$ and 4.5 , respectively. This 3-D plot is similar to the 3-D plot of 
energy versus $H_{\text {in }}$ and angle of fcc ferromagnetic film with four layers except the position of spikes. Here the other magnetic energy parameters were kept at $\omega=1$, $D_{m}{ }^{(2)}=30, D_{m}{ }^{(4)}=20, J=10, K_{s}=H_{\text {out }}=\frac{N_{d}}{\mu_{0}}=10$

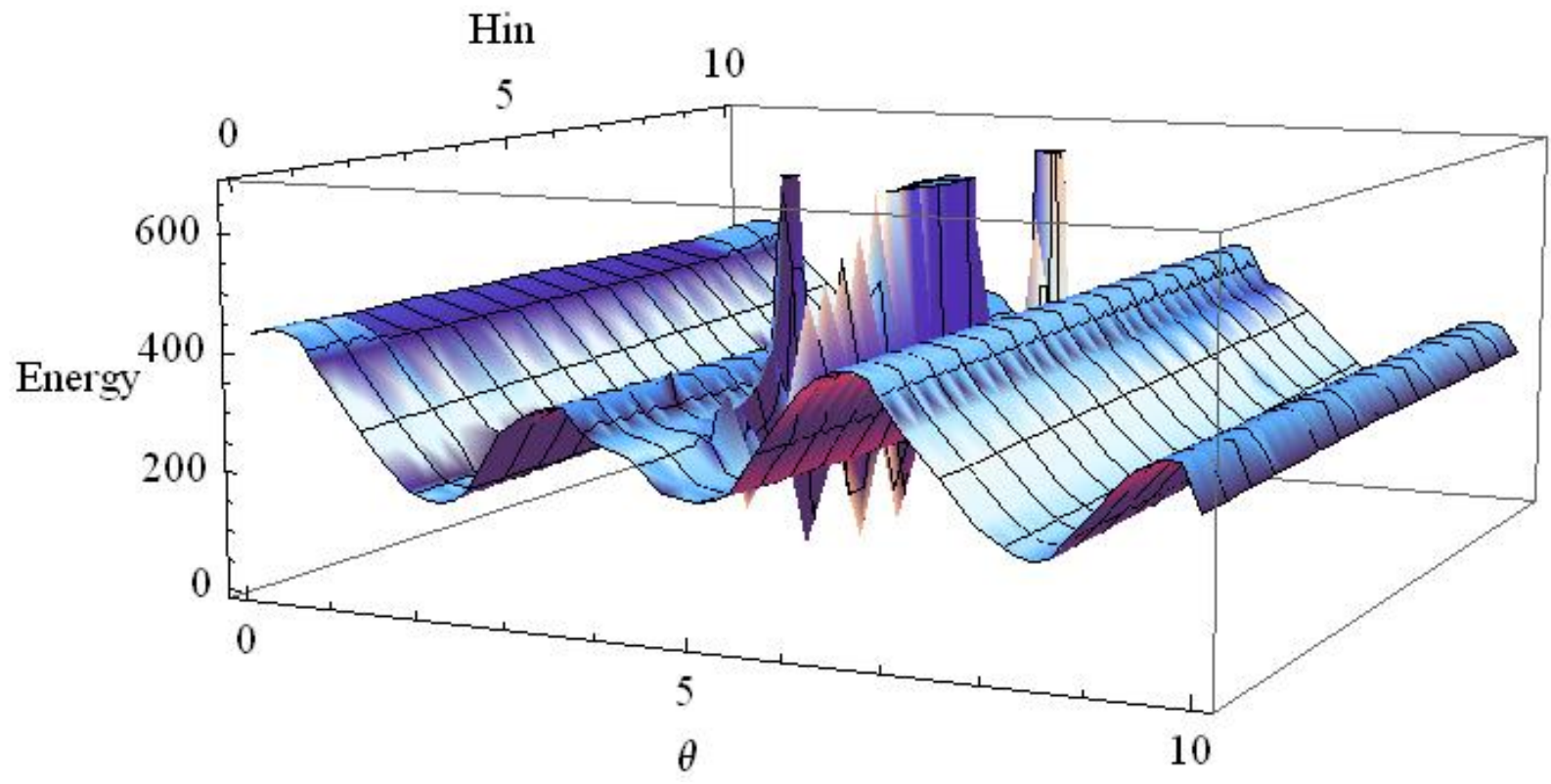

Figure 5: 3-D plot of energy versus angle and $H_{\text {in }}$ for film with five layers

\section{CONCLUSIONS}

The 3-D plots of fcc ferromagnetic films with four spin layers are similar to those of ferromagnetic films with five layers plotted for the same magnetic energy parameters. However, the positions and number of spikes are different in two plots. Spikes occur due to the singularities in the equation of the total energy. The angle between the magnetic easy and hard directions was found to be 97 degrees according to all the graphs plotted for four and five layers. The theoretical value of this angle is fairly close to the experimental value. The magnetic easy axis rotates toward the in plane direction with the increase of the number of layers. This result also agrees with the experimental data of spin reorientation transition obtained for sputter synthesized fcc structured ferromagnetic Ni films and Fe films prepared using electron beam evaporation method. 


\section{REFERENCES}

Araya-Pochet J., Ballentine C.A., Erskine J.L., 1988. Thickness and temperature dependent spin anisotropy of ultrathin epitaxial Fe films on $\mathrm{Ag}(100)$. Physical Review B 38(11), 7846-7849. http://dx.doi.org/10.1103/PhysRevB.38.7846

Dantziger M., Glinsmann B., Scheffler S., Zimmermann B. and Jensen P.J., 2002. In-plane dipole coupling anisotropy of a square ferromagnetic Heisenberg monolayer. Physical Review B 66, 094416. http://dx.doi.org/10.1103/PhysRevB.66.094416

Ernst A., Lueders M., Temmerman W.M., Szotek Z. and Van der Laan G., 2000. Theoretical study of magnetic layers of nickel on copper; dead or live?. Journal of Physics: Condensed matter 12(26), 5599-5606. http://dx.doi.org/10.1088/0953-8984/12/26/306

Hucht A. and Usadel K.D., 1997. Reorientation transition of ultrathin ferromagnetic films. Physical Review B 55, 12309. http://dx.doi.org/10.1103/PhysRevB.55.12309

Hucht A. and Usadel K.D., 1999. Theory of the spin reorientation transition of ultra-thin ferromagnetic films. Journal of Magnetism and Magnetic materials 203(1), 88-90. http://dx.doi.org/10.1016/S0304-8853(99)00197-3

Kovachev St and Wesselinowa J.M., 2009. Theoretical study of multiferroic thin films based on a microscopic model. Journal of Physics: Condensed matter 21(22), 225007. http://dx.doi.org/10.1088/0953-8984/21/22/225007

Nowak U., 1995. Magnetisation reversal and domain structure in thin magnetic films: Theory and computer simulation. IEEE transaction on magnetics 31(6-2), 4169-4171.

Parlak U., Akoz M.E., Tokdemir Ozturk S. and Erkovan M., 2015. Thickness dependent magnetic properties of polycrystalline nickel thin films. Acta Physica Polonica A 127(4), 995-997. http://dx.doi.org/10.12693/APhysPolA.127.995

Radomska Anna and Balcerzak Tadeusz, 2003. Theoretical studies of model thin EuTe films with surface elastic stresses. Central European Journal of Physics 1(1), 100-117.

Samarasekara P. and Cadieu F.J., 2001. Magnetic and Structural Properties of RF Sputtered Polycrystalline Lithium Mixed Ferrimagnetic Films. Chinese Journal of Physics 39(6), 635640.

Samarasekara P., 2002. Easy Axis Oriented Lithium Mixed Ferrite Films Deposited by the PLD Method. Chinese Journal of Physics 40(6), 631-636.

Samarasekara P., 2003. A Pulsed RF Sputtering Method for Obtaining Higher Deposition Rates. Chinese Journal of Physics 41(1), 70-74.

Samarasekara P., 2006. Second order perturbation of Heisenberg Hamiltonian for non-oriented ultrathin ferromagnetic films. Electronic Journal of Theoretical Physics 3(11), 71-83.

Samarasekara P., 2006. A solution of the Heisenberg Hamiltonian for oriented thick ferromagnetic films. Chinese Journal of Physics 44(5), 377-386.

Samarasekara P., 2007. Classical Heisenberg Hamiltonian solution of oriented spinel ferrimagnetic thin films. Electronic Journal of Theoretical Physics 4(15), 187-200.

Samarasekara P. and De Silva S.N.P., 2007. Heisenberg Hamiltonian solution of thick ferromagnetic films with second order perturbation. Chinese Journal of Physics 45(2-I), 142-150. 
Samarasekara P., 2010. Determination of energy of thick spinel ferrite films using Heisenberg Hamiltonian with second order perturbation. Georgian electronic scientific journals: Physics $1(3), 46-49$.

Samarasekara P., 2011. Investigation of Third Order Perturbed Heisenberg Hamiltonian of Thick Spinel Ferrite Films. Inventi Rapid: Algorithm Journal 2(1), 1-3.

Samarasekara P. and William A. Mendoza, 2011. Third Order Perturbed Heisenberg Hamiltonian of Spinel Ferrite Ultra-thin films. Georgian electronic scientific journals: Physics 1(5), 15-24.

Tsai Shan-Ho, Landau D.P. and Schulthess Thomas C., 2003. Effect of interracial coupling on the magnetic ordering in ferro-antiferromagntic bilayers. Journal of Applied Physics 93(10), 86128614. http://dx.doi.org/10.1063/1.1557794

Usadel K.D. and Hucht A., 2002. Anisotropy of ultrathin ferromagnetic films and the spin reorientation transition. Physical Review B 66, 024419. http://dx.doi.org/10.1103/PhysRevB.66.024419

Zhao D., Feng Liu, Huber D.L. and Lagally M.G., 2002. Step-induced magnetic-hysteresis anisotropy in ferromagnetic thin films. Journal of Applied Physics 91(5), 3150-3153. http://dx.doi.org/10.1063/1.1433179 